\title{
A Profile Hidden Markov Model to investigate the distribution and frequency of LanB-encoding lantibiotic modification genes in the human oral and gut microbiome
}

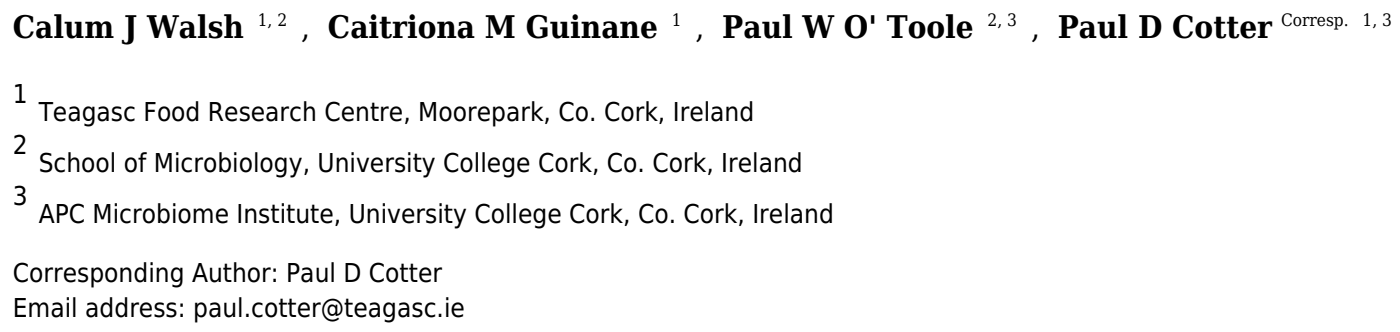

The human microbiota plays a key role in health and disease, and bacteriocins, which are small, bacterially produced, antimicrobial peptides, are likely to have an important function in the stability and dynamics of this community. Here we examined the density and distribution of the subclass I lantibiotic modification protein, LanB, in human oral and stool microbiome datasets using a specially constructed profile Hidden Markov Model (HMM).

The model was validated by correctly identifying known lanB genes in the genomes of known bacteriocin producers more effectively than other methods, while being sensitive enough to differentiate between different subclasses of lantibiotic modification proteins. This approach was compared with two existing methods to screen both genomic and metagenomic datasets obtained from the Human Microbiome Project (HMP).

Of the methods evaluated, the new profile HMM identified the greatest number of putative LanB proteins in the stool and oral metagenome data while BlastP identified the fewest. In addition, the model identified more LanB proteins than a pre-existing Pfam lanthionine dehydratase model. Searching the gastrointestinal tract subset of the HMP reference genome database with the new HMM identified seven putative subclass I lantibiotic producers, including two members of the Coprobacillus genus.

These findings establish custom profile HMMs as a potentially powerful tool in the search for novel bioactive producers with the power to benefit human health, and reinforce the repertoire of apparent bacteriocin-encoding gene clusters that may have been overlooked by culture-dependent mining efforts to date. 
1 Author Cover Page

2 A Profile Hidden Markov Model to investigate the distribution and frequency of LanB-encoding lantibiotic modification genes in the human oral and gut microbiome

4

5 Calum J. Walsh ${ }^{1,3}$, Caitriona M. Guinane ${ }^{1}$, Paul W. O’Toole ${ }^{2,3}$, Paul D. Cotter ${ }^{1,2 *}$

6

7 Teagasc Food Research Centre, Moorepark, Fermoy, Cork, Ireland ${ }^{1}$;

8 APC Microbiome Institute, University College Cork, Cork, Ireland²;

9 School of Microbiology, University College Cork, Cork, Ireland ${ }^{3}$.

*To whom correspondence should be addressed: paul.cotter@teagasc.ie

12

13

14

15

16

17 
19 Abstract

20 Background

21 The human microbiota plays a key role in health and disease, and bacteriocins, which are small,

22 bacterially produced, antimicrobial peptides, are likely to have an important function in the

23 stability and dynamics of this community. Here we examined the density and distribution of the

24 subclass I lantibiotic modification protein, LanB, in human oral and stool microbiome datasets

25 using a specially constructed profile Hidden Markov Model (HMM).

26 Methods

27 The model was validated by correctly identifying known lanB genes in the genomes of known

28 bacteriocin producers more effectively than other methods, while being sensitive enough to

29 differentiate between different subclasses of lantibiotic modification proteins. This approach was

30 compared with two existing methods to screen both genomic and metagenomic datasets obtained

31 from the Human Microbiome Project (HMP).

32 Results

33 Of the methods evaluated, the new profile HMM identified the greatest number of putative LanB

34 proteins in the stool and oral metagenome data while BlastP identified the fewest. In addition,

35 the model identified more LanB proteins than a pre-existing Pfam lanthionine dehydratase

36 model. Searching the gastrointestinal tract subset of the HMP reference genome database with

37 the new HMM identified seven putative subclass I lantibiotic producers, including two members

38 of the Coprobacillus genus. 
39

40

41

42

43

44

45

46

47

48

49

50

51

52

53

54

55

\section{Conclusions}

These findings establish custom profile HMMs as a potentially powerful tool in the search for novel bioactive producers with the power to benefit human health, and reinforce the repertoire of apparent bacteriocin-encoding gene clusters that may have been overlooked by culturedependent mining efforts to date.

\section{Background}

Bacteriocins are ribosomally synthesised peptides produced by bacteria that inhibit the growth of other bacteria. Some classes of bacteriocins are post-translationally modified to provide structures beyond those possible by ribosomal translation alone. These modifications are typically key to the peptide's functionality, stability and target recognition (Arnison et al. 2013). Class I bacteriocins, also known as lantibiotics, are one such class of small $(<5 \mathrm{kDa})$ modified bacteriocins, possessing the characteristic thioester amino acids lanthionine or methyllanthionine (Perez et al. 2014). Lantibiotics form a subgroup within the larger lantipeptide family, which also includes peptides that lack antimicrobial activity. Lantipeptides can be divided into four different subclasses (I - IV) based on the distinct biosynthetic enzymes responsible for their posttranslational modification (Arnison et al. 2013).

5 The most commonly studied lantibiotic, Nisin, is a subclass I lantibiotic, meaning that the linear prepeptide is processed by a LanBC modification system (Arnison et al. 2013). The core peptide undergoes a two-step posttranslational modification catalysed by two distinct enzymes - the dehydratase LanB and the cyclase LanC (Xie \& van der Donk 2004). The leader sequence, necessary for recognition by the modification enzymes in the two previous steps, is then removed by the protease LanP to produce the active lantibiotic (Xie \& van der Donk 2004). The 
61 gene-encoded nature of bacteriocins and bacteriocin-like peptides makes them ideal candidates

62 for genome mining. In the case of modified bacteriocins, the structural prepeptide coding

63

sequence often appears alongside the genes encoding proteins responsible for its modification and export from the cell. However, as more bacteriocins are discovered, the heterogeneous nature of these prepeptides is becoming ever more apparent. This diversity, coupled with their small sequence length, makes bacteriocin prepeptides much more difficult to detect using sequence-homology based searches like BLAST (Altschul et al. 1990). In an effort to address these obstacles, shifting the focus to the detection of bacteriocin-associated proteins opens up more avenues of discovery than simply searching for prepeptide homologs. This provides opportunities to better determine the frequency with which specific types of bacteriocin gene clusters can be found in different environmental niches, such as the human microbiota, through the investigation of metagenomic data.

It has been estimated that the human microbiota comprises approximately 100 trillion bacterial cells, outnumbering our own cells by a factor of 10 or more (Bäckhed et al. 2005). A recent publication, however, has argued that the ratio is actually more likely to be one-to-one, with the numbers being similar enough that each defecation event may alter the ratio to favour human cells over bacteria (Sender et al. 2016). Of greater consequence than bacterial numbers, however, is the collection of genes encoded in this metagenome, thought to be approximately 150 times larger than that of the human genome, with a functional potential far broader than that of its host (Qin et al. 2010). Regardless of absolute numbers, this dynamic community is thought to contain 100-1000 phylotypes (Faith et al. 2013; Qin et al. 2010) and play an integral role in human health and disease (Clemente et al. 2012; Flint et al. 2012). The human microbiota exhibits robust temporal stability (Belstrøm et al. 2016; Jeffery et al. 2016) perhaps due, in part, to the 
84 protection against invading bacteria conferred by bacteriocins and other antimicrobials produced

85 in situ (Corr et al. 2007; Moroni et al. 2006; Rea et al. 2011a). As such, investigation of the

86 density and diversity of bacteriocins produced in the microbiome of healthy individuals may

87 shed light on beneficial and harmful members of this community, and key organisms for maintaining typical, i.e. health-associated, microbiota composition.

Mining the human microbiota, especially for antimicrobial compounds, has become a popular area of research in recent years (Donia et al. 2014; Walsh et al. 2015). Due to the availability of metagenomic data generated by large public funding initiatives such as the Human Microbiome Project in the U.S. (The Human Microbiome Project Consortium 2012) and the European MetaHIT consortium (Dusko Ehrlich 2010), in silico mining of data has emerged as a new tool that has the potential to identify antimicrobial-producing probiotics that can modulate the gut microbiota (Erejuwa et al. 2014; Walsh et al. 2014), or address the increasingly serious threat to public health caused by antimicrobial resistance. There are many available tools for mining the microbiome for antimicrobials, including BAGEL3 (van Heel et al. 2013), antiSMASH (Weber et al. 2015), and traditional sequence-based approaches like BLAST (Altschul et al. 1990). A feature commonly integrated into these tools are Hidden Markov Models (HMM) (Morton et al. 2015; van Heel et al. 2013; Weber et al. 2015), a statistical method often used to model biological data such as speech recognition, disease interaction and changes in gene expression in cancer (Gales \& Young 2007; Seifert et al. 2014; Sherlock et al. 2013). Profile HMMs, a specific subset of HMMs, represent the patterns, motifs and other properties of a multiple sequence alignment by applying a statistical model to estimate the true frequency of a nucleotide or amino 105 acid at a given position in the alignment from its observed frequency (Yoon 2009). Profile HMMs differ from general HMMs as they move strictly from left to right along the alignment 
107 and do not contain any cycles, a feature that makes them suitable for modelling nucleotide and

108 protein sequence data, and have been notably utilized to detect viral protein sequences in

109 metagenomic sequence data (Skewes-Cox et al. 2014). For each column in the multiple sequence

110 alignment, the profile uses one of three types of hidden state - a match state, an insert state, or a

111 delete state, to describe residue frequencies, insertions, and deletions, respectively (Yoon 2009).

112 Profile HMMs are potentially more sensitive than sequence homology approaches for identifying

113 more distantly related proteins as they focus on function-dependent conserved motifs that are

114 theoretically slower-evolving, as opposed to focusing on overall sequence similarity. Indeed,

115 profile HMMs are known to typically outperform pairwise sequence comparison methods (such

116 as BLAST) in the detection of distant homologs (Park et al. 1998), at the cost of greater

117 computational requirements - particularly in alignment scoring and E-value calculation (Madera

118 \& Gough 2002). Correspondingly, speed is the main advantage of BLAST over profile HMMs,

119 however, as it is a heuristic algorithm it does not guarantee identification of the optimal

120 alignment between query and database sequences.

121 In this study we designed, validated and implemented a Profile HMM to search for putative

122 subclass I lantibiotic gene clusters in the HMP metagenomes and compared its performance to

123 some of the tools mentioned above.

124 Methods

\section{Data Collection}

126 HMASM (HMP Illumina WGS Assemblies) and HMRGD (HMP Reference Genomes Data)

127 were downloaded from the Data Analysis and Coordination Centre for the HMP. 835 bacterial 
128 RefSeq protein sequences annotated as "lantibiotic dehydratase" were downloaded from NCBI

129 Protein website (13 Apr 2015) in FASTA format (listed in Supplemental Table 1).

130

131

132

133

134

135

136

137

138

139

140

141

142

143

144

145

146

147

148

149

\section{Building and Validating the new Profile Hidden Markov Model}

A global multiple sequence alignment was generated in the aligned-FASTA format using MUSCLE (v3.8.31) (Edgar 2004), and a profile HMM was built from the MSA aligned-FASTA file using the HMMER tool hmmbuild (v3.1b1 May 2013). For comparison of the new model's performance, HMMER3's hmmsearch tool was used, with default parameters, to search the Pfam lantibiotic dehydratase model PF04738 against the same stool and oral HMASM assemblies (the sequences used to build this model are listed in Supplemental Table 2). Positive and negative controls (listed in Table 1) were used to evaluate the model's ability to 1) accurately identify LanB protein sequences, and 2) distinguish LanB protein sequences from other, related, lantibiotic modification proteins (i.e. LanM, LanKC, and LanL). The controls were also screened using the PF04738 model, the web-based bacteriocin genome mining tool BAGEL3 (van Heel et al. 2013), and a traditional BlastP using the nisin-associated lanthionine dehydratase, NisB, as the driver sequence (GenBank accession number CAA79468.1) to compare the sensitivity and specificity of each approach. A flowchart of the steps involved in building, validating and applying a profile HMM is depicted in Supplemental Figure 1.

\section{Target Sequence Translation}

The HMMER3 hmmsearch tool only accepts protein sequences as targets for comparison to protein profile HMMs so a python script was created to translate the nucleotide sequences into protein sequences. The DNA nucleotide sequences were translated in six frames using the standard genetic code. 


\section{Metagenomic Screen}

151 The HMMER3 tool hmmsearch was used, with default parameters, to search both the new LanB

152 profile HMM and the Pfam PF04738 profile HMM (Punta et al. 2012) against the stool and oral

153 subsets of the Human Microbiome Project's whole metagenomic shotgun sequencing assemblies

154 (HMASM). 139 stool communities and 382 communities from eight different body sites within

155 the oral cavity were screened from the HMP database. These are listed in Table 2. As an

156 additional comparison of performance, a traditional BlastP screen was performed on the same

157 metagenomic samples using the previously mentioned nisin-associated lanthionine dehydratase,

158 NisB, driver sequence. A significance cutoff of $\mathrm{E} \leq 1 \times 10^{-5}$ was chosen for both profile HMM

159 and BlastP methods.

\section{Manual Examination of Randomly Selected Gene Neighbourhoods}

161 A subset of sixty hits were randomly selected and the surrounding region examined to identify

162 other proteins involved in lantibiotic biosynthesis. Open Reading Frames were identified using

163 Glimmer v3.02 (Delcher et al. 1999), which were then visualised using Artemis (Carver et al.

164 2012) and blasted against the nr database using BlastP.

\section{Genomic Screen}

166 HMMER3's hmmsearch tool was used, with default parameters, to search the new profile HMM

167 against the draft genomes comprising the gastrointestinal tract subset of the Human Microbiome

168 Project's reference genome database. 
169 Taxonomic Classification of LanB-encoding contigs

170 Taxonomy was assigned to LanB-encoding contigs, as assigned by our pHMM, using Kaiju

171 (Menzel et al. 2016). Analysis was performed in MEM run mode using default parameters and

172 the NCBI non-redundant protein database.

\section{Statistical Analysis}

174 Statistical analysis was performed in R (v. 3.1.3) (R Core Team 2015).

175 Results

\section{Validation of the Profile Hidden Markov Model}

177 The ability of the newly developed profile HMM and the Pfam lantibiotic dehydratase model

178 PF04738 to detect LanB-encoding genes were compared using the positive and negative controls

179 listed in Table 1. The positive controls were selected based on a relevant book chapter (Rea et al.

180 2011b) and all are previously characterised bacteriocin producers for which the sequence of the

181 relevant biosynthetic gene cluster was available. None of the positive control sequences were

182 used in the building of the model and a graphical representation of these clusters is presented in

183 Figure 1. Lactococcus lactis subsp. lactis KF147 was chosen as a negative control because it is

184 of the same subspecies as three of the positive controls (Lactococcus lactis subsp. lactis S0,

185 Lactococcus lactis subsp. lactis CV56 and Lactococcus lactis subsp. lactis IO-1) but does not

186 produce a bacteriocin. Streptococcus mutans GS-5, Streptomyces cinnamoneus cinnamoneus

187 DSM 4005, the Lactococcus lactis subsp. lactis IL1835 plasmid pES2, the Streptococcus

188 salivarious plasmid pSsal-K12, and the newly characterised formicin producer Bacillus

189 paralicheniformis APC 1576 were chosen as negative controls to evaluate the ability of the

190 model to differentiate between LanB (subclass I) proteins and the LanM proteins-from these 
191 strains, which perform a similar, but distinct, function in the posttranslational modification of

192 subclass II lantibiotics. Streptomyces venezuelae ATCC 10712 was chosen as the final negative

193 control as it has been reported to produce a LanL-type lantipeptide (Goto et al. 2010).

194 Examination of the ATCC 10712 genome using BAGEL3 identified several other orphan

195 lantibiotic modification genes, including those encoding putative LanL, LanM, LanD and LanB

196 proteins. The genome also appeared to encode a subclass III lantipeptide cluster comprised of

197 genes potentially encoding a structural protein, two ABC-type transporters and a LanKC

198 modification protein. Notably, there have been no reports of subclass I lantibiotic production by

199 ATCC 10712 despite an in-depth investigation into the strain's lantipeptide producing capability

200 (Goto et al. 2010), and BAGEL3 identified no other lantibiotic-related genes in the area of

201 interest leading us to determine that this was a false positive.

202 The newly developed LanB profile HMM correctly identified the LanB protein in all nine

203 positive controls, while the PF04738 profile HMM correctly identified the LanB protein in eight

204 of the nine positive controls, failing to detect the Bsa-associated LanB protein in Staphylococcus

205 aureus subsp. aureus USA300_FPR3757. Both the LanB and PF04738 profile HMMs returned

206 no false positives when searched against the seven negative controls used in this study.

207 The web version of BAGEL3 correctly identified the lantibiotic modification proteins in all

208 positive and negative controls, excepting the aforementioned ATCC 10712-encoded LanB

209 concluded to be a false positive. Interestingly, examination of these controls with the BlastP

210 method described previously, failed to correctly identify the LanB proteins encoded by

211 Streptococcus mutans $\mathrm{CH} 43$ and Streptococcus mutans UA787, although the former $\left(\mathrm{E}=3 \times 10^{-4}\right)$

212 fell just short of the significance cutoff $\left(E \leq 1 \times 10^{-5}\right)$. BlastP also incorrectly identified a LanB

213 protein in the negative control Lactococcus lactis subsp. lactis KF147. 


\section{Metagenomic Screen}

215 A search with the newly developed profile HMM against the HMASM database identified 399

216 hits from the stool metagenomes and 1169 hits from the oral metagenomes. In contrast, the

217 PF04738 model identified 288 hits from the stool metagenomes and 686 from the oral

218 metagenomes. Our model reported at least one putative lantibiotic gene cluster in $81 \%$ of oral

219 metagenomes and $86 \%$ of stool metagenomes, compared to $73 \%$ and $76 \%$, respectively,

220 identified by the Pfam model. The distribution of hits per sample is presented in Figure 2. BlastP

221 identified 231 hits from the stool metagenomes and 374 hits from the oral metagenomes. The

222 results of these three approaches were compared to ascertain what proportion of significant hits

223 was common to more than one search method. The results of this comparison are summarised in

224 Figure 3 and show that the newly developed profile HMM identified the greatest number of

225 lantibiotic modification genes in datasets from both body sites, while the BlastP approach

226 identified the fewest.

227 The overall results of these combined screening approaches, illustrated in Figure 4 and

228 summarised in Supplemental Table 3, show a higher number and density of hits in the oral

229 metagenomes than in the stool metagenomes (Welch's two sample t-test, $p=5.422 \mathrm{e}-08$ ) and they

230 also reveal a large variation in density of hits between the different sites within the oral

231 metagenomes. This pattern was also reflected in four of the Oral subsites, namely Buccal

232 Mucosa, Subgingival Plaque, Supragingival Plaque and Tongue Dorsum, all of which had a

233 significantly higher LanB density than the Stool metagenomes $(p=0.00304,0.02467,5.5 \mathrm{e}-08$,

234 and 0.00024 , respectively). Within the Oral samples, our model revealed a large variation in

235 density of hits between different subsites. The throat metagenomes had the lowest LanB density,

236 and exhibited significantly lower densities than Buccal Mucosa $(p=0.00581)$, Subgingival 
237 Plaque $(p=0.00587)$, Supragingival Plaque $(p=0.00943)$, and Tongue Dorsum $(p=0.02219)$

238 subsites.

239

240

241

242

243

244

245

246

247

248

249

250

251

252

253

254

255

256

257

258

\section{Manual Examination of Selected Gene Neighbourhoods}

Sixty hits, listed in Supplemental Table 4, were randomly selected from those identified by the new profile HMM, 45\% (27/60) of which were identified by at least one of the other two

methods, and manually examined to determine if a bacteriocin gene cluster could be identified. $42 \%(25 / 60)$ of these were not further analysed because the often relatively short regions assembled from the shotgun data prevented the identification of a full lantibiotic gene cluster. However, of the 35 remaining clusters, $28(80 \%)$ appeared to encode multiple genes involved in the biosynthesis of bacteriocins and thiopeptides. These genes encode proteins involved in posttranslational modification, bacteriocin transport, leader cleavage and regulation (Supplemental Figure 2).

81 hits identified by BlastP were missed by both profile HMM approaches. 50 of these originated in the stool metagenomes and were selected for manual annotation to determine if an overall structure or similarity could be observed. 29 of these 50 were part of clusters whose components showed relatively low sequence identity (39-50\%) with proteins responsible for the biosynthesis of thiopeptides and lantibiotics, including a putative lanthionine dehydratase, a radical SAM/SPASM domain-containing protein, a thiopeptide-type bacteriocin biosynthesis domaincontaining protein, an S41 family peptidase, and a protein of unknown function (DUF4932) predicted to be a putative metalloprotease. All 50 manually annotated gene clusters are available in GENBANK format and an example of this cluster architecture is summarised in Supplemental Table 5. 
259

260

261

262

263

264

265

266

267

268

269

270

271

272

273

274

275

276

277

278

279

280

\section{Genomic Screen}

The draft genomes of the gastrointestinal tract subset of the HMRGD were also used as a database and searched using the new profile HMM. This resulted in the identification of seven hits, including two strains of Coprobacillus, a potentially probiotic genus (Stein et al. 2013; Yan et al. 2012) (Table 3). From these seven genomes, only three lantibiotic gene clusters were identified by BAGEL3, these are illustrated in Figure 5. Although this low frequency of lanthionine dehydratase proteins in the genomic dataset $(0.006$ hits/Mb) contrasts with the findings of the metagenome screen reported above, it is in agreement with previous reports of relatively low subclass I lantibiotic density within the human microbiota (Walsh et al. 2015; Zheng et al. 2014). A possible explanation for this significantly lower gene density (Welch's two sample t-test, $p=1.232 \mathrm{e}-10$ ) is that the subclass I lantibiotic clusters identified in the metagenomic data by the new profile HMM are present in the genomes of rarer members of the gut microbiota, which are not represented in the HMP reference genome database.

\section{Taxonomic Classification of LanB-encoding contigs}

The MEM run mode of Kaiju works by searching for exact matches of given length between the query and database sequences, in the case of multiple hits of the same length in different taxa, a lowest common ancestor is inferred. Kaiju classified 378 of 399 LanB-encoding contigs. Of these, 232 were classified to the species level - however 68 were removed as their exact species was ambiguous. Of the remaining 164 classified contigs, $66(40.2 \%)$ were represented at the species-level in the previously screened HMRGD database. The most abundant genus was Alistipes, accounting for $14.03 \%$ of LanB-encoding cotigs identified by our model, followed by Blautia (7.77\%), Clostridium (4.51\%), and Bacteroides (3.76\%) (Supplemental Table 6). 
281

282

283

284

285

286

287

288

289

290

291

292

293

294

295

296

297

298

299

300

301

302

303

\section{Discussion}

Bacteriocin production enhances the competitiveness of bacteria living in complex communities and has the potential to be harnessed for the benefit of human health. The goal of this study was to develop a profile HMM and to assess its ability, in comparison with several other approaches, to detect putative subclass I lantibiotic gene clusters in human metagenomic datasets. Through this process it was also possible to evaluate the potential frequency and distribution of these bacteriocin gene clusters in the human microbiota.

To validate the model, nine positive controls and five negative controls were selected to evaluate its sensitivity and specificity. These controls were selected based on reported bacteriocin production; all positive controls were known producers of subclass I lantibiotics while the negative controls produced either different subclasses of lantibiotics or none at all. Following validation, genomic and metagenomic data corresponding to two niches within the human microbiome were chosen as the focus of this study. The first of these niches was human stool and was selected as the corresponding samples were most likely to yield bacteriocin producers with the potential to modulate undesirable microbiota profiles associated with obesity, colorectal cancer, type 2 diabetes or inflammatory bowel diseases due to their ability to survive and colonise this environment. Secondly, human oral communities were examined as a previous study showed that they contained, by far, the greatest proportion of bacteriocin structural genes across a number of human metagenome samples (Zheng et al. 2014). Zheng et al. reported that $80 \%$ of class I bacteriocins (lantibiotics) and $89 \%$ of all bacteriocins identified using their method originated in the oral metagenomes, while the stool metagenomes contained just $15 \%$ and $7 \%$, respectively. The same study reported that $88 \%$ of samples from the oral cavity and $73 \%$ of samples from the gut contained at least one bacteriocin (regardless of class), while the 
304 new profile HMM reported these statistics as $81 \%$ and $83 \%$, respectively for subclass I

305 lantibiotics alone. The in silico screen carried out with the profile HMM is consistent with the

306 observation by Zheng et al. (Zheng et al. 2014) by yielding a higher number and density of hits

307 from the oral, compared to the stool, metagenomic data. Furthermore, the large variation in

308 density of hits between sites within the oral environment suggests that lantibiotic production

309 confers a greater advantage in buccal musoca, subgingival plaque, supragingival plaque, and

310 tongue dorsum communities compared to communities from the throat. This may be due to the

311 direct benefits of antimicrobial activity but could also involve the intra- and interspecies

312 signalling roles attributed to lantibiotic peptides (Upton et al. 2001), particularly in the intensely

313 competitive microbial biofilm environment of dental plaque.

314 One of the most interesting observations from the study was the large variation in the numbers of

$315 \operatorname{lan} B$ genes reported by the three different approaches. The BlastP approach identified, by far, the

316 lowest number of significant hits overall and the lowest in every body site examined, except for

317 the saliva microbiome. Our model identified more than double the number of hits provided by

318 the BlastP-based approach, in line with the aforementioned knowledge that profile HMMs can

319 detect as much as three times as many distant homologs than pairwise methods (Park et al.

320 1998). Our model also identified a greater number of LanB proteins than the Pfam PF04738

321 model when used to search the same data using the same parameters. While the PF04738 model

322 relates to the $\mathrm{C}$-terminus of the lanthionine dehydratase protein, responsible for the glutamate

323 elimination step of lantibiotic modification (Ortega et al. 2015), the newly developed profile

324 HMM takes the full length of the LanB protein into consideration, thereby providing greater

325 predictive power. Our model, in addition to identifying more potential LanB proteins, also

326 exhibited greater sensitivity and specificity during validation than all other methods used to 
327 analyse the controls. As stated above, profile HMMs are already known to be particularly

328 sensitive, the validation step, however, also suggests that they are more specific than the other

329 methods evaluated as they were the only approach which did not return any false positives.

330 When selecting the controls used to examine the performance of the different approaches, greater

331 consideration was given to the quality of these controls than their quantity. Only controls with

332 experimentally characterised lantibiotic production were included in the validation dataset. This

333 relatively small control group means that, although the results of the validation step may explain

334 the contrasting numbers of LanB proteins reported by our model and the PF04738 model, it

335 cannot be said for certain that our model performed better.

336 Zheng et al., using the same metagenomic data that was the focus of this study, identified 17

337 potential subclass I lantibiotics from stool samples and 76 from oral samples, a much lower

338 frequency of detection than in this study, probably due to the different methodologies used. That

339 study focused on searching for proteins similar to those in BAGEL3's manually curated

340 database, an approach which likely lost sensitivity because bacteriocin precursor peptides can

341 differ considerably at primary sequence level. Furthermore, the screen employed a BLAST-

342 based approach which, as demonstrated here, exhibited the lowest number of significant hits

343 reported.

344 To investigate the areas surrounding the LanB-encoding genes identified by our model we

345 randomly selected thirty positive hits from the oral and stool metagenome screens for manual

346 examination. This approach revealed that several of the hits were on scaffolds that were either

347 too small to contain a full gene or did not contain the gene's start codon. This was most likely as

348 a consequence of the fragmented nature of the metagenomic data, as opposed the identification

349 of true false positives by the model and would probably occur regardless of the method 
350 employed. 42\% (25/60) of hits selected for manual examination were discarded based on these

351 criteria. It also revealed that a considerable number of hits exhibited low $(\sim 30 \%)$ similarity to

352 putative thioesterases in the nr protein sequence database, highlighting that lanthionine

353 dehydratases are relatively-closely related to proteins involved in the posttranslational

354 modification of thiopeptides, most likely those responsible for dehydration of serine and

355 threonine residues (Garg et al. 2013). The similarity between these dehydratase proteins suggests

356 a possible common ancestor protein (Kelly et al. 2009). Another possible explanation relates to

357 the fact that all of the proteins annotated as thiopeptide modification proteins are putative

358 annotations and none, to our knowledge, have been confirmed as such in vitro. It is possible,

359 therefore, that these may simply be lanthionine dehydratases which have been incorrectly

360 annotated due to automatic software and incomplete/under-curated databases. The majority of

361 clusters identified contained genes encoding both LanB and LanC modification proteins, while

362 many also contained a leader cleavage and activation peptidase and/or ABC transporter proteins

363 for export of the mature peptide, suggesting that these have the potential to encode a functional

364 lantibiotic.

365 To evaluate the model's performance in a genomic context we applied it to the gastrointestinal

366 tract subset of the HMP's reference genome database and compared the results to our previously

367 published study which used the online bacteriocin genome mining tool BAGEL3 to screen this

368 same database (Walsh et al. 2015). The results of the two screens were startlingly different and

369 served to highlight the variation in results that can arise from applying different methods to the

370 same data. Interestingly, the gastrointestinal tract reference genomes encoded a significantly

371 lower frequency of LanB hits than the stool metagenomic samples. Taxonomic classification of

372 the 399 LanB-encoding contigs identified by our new model from the stool metagenomes 
373 revealed that only $40.2 \%$ of these potential lantibiotic producing strains were represented in the

374 reference genome database, suggesting that the majority of these lantibiotics were encoded by

375 species rarer members of the gut microbiota or those that have not previously been identified as

376 important. Taxonomic classification of these LanB-encoding contigs also served to highlight

377 patterns in the results of the three approaches used (Supplemental Figure 3), for example out

378 model identified Allokutzneria, Coprococcus, Enterovibrio, Paenibacillus, and Tenicibaculum-

379 encoded LanB proteins that were completely missed by the Pfram and BlastP approaches.

\section{Conclusions}

381 Across the oral and stool communities examined, this study identified 2007 unique putative

382 subclass I lantibiotic biosynthetic gene clusters by three different methods, further emphasising

383 the tremendous potential that the human microbiota has as a source of therapeutic compounds.

384 As this study was performed entirely in silico, the next challenge lies in experimentally

385 identifying and characterising these putative bacteriocins to identify those with the ability to

386 desirably modulate the microbiota for the treatment of disease.

\section{Acknowledgements}

388 The authors would like to thank Manimozhiyan Arumugam for helpful discussion.

List of Abbreviations

Abbreviation Description

HMASM Human Microbiome Project's Illumina Whole Genome Shotgun Assemblies

HMM Hidden Markov Model 
HMP Human Microbiome Project

HMRGD Human Microbiome Project's Reference Genome Data

\section{References}

Hmmer. Available at http://hmmer.org.

Human Microbiome Project; Data Analysis and Coordination Center. Available at http://hmpdacc.org/.

Altschul SF, Gish W, Miller W, Myers EW, and Lipman DJ. 1990. Basic local alignment search tool. J Mol Biol 215:403-410. 10.1016/s0022-2836(05)80360-2

Arnison PG, Bibb MJ, Bierbaum G, Bowers AA, Bugni TS, Bulaj G, Camarero JA, Campopiano DJ, Challis GL, Clardy J, Cotter PD, Craik DJ, Dawson M, Dittmann E, Donadio S, Dorrestein PC, Entian K-D, Fischbach MA, Garavelli JS, Goransson U, Gruber CW, Haft DH, Hemscheidt TK, Hertweck C, Hill C, Horswill AR, Jaspars M, Kelly WL, Klinman JP, Kuipers OP, Link AJ, Liu W, Marahiel MA, Mitchell DA, Moll GN, Moore BS, Muller R, Nair SK, Nes IF, Norris GE, Olivera BM, Onaka H, Patchett ML, Piel J, Reaney MJT, Rebuffat S, Ross RP, Sahl H-G, Schmidt EW, Selsted ME, Severinov K, Shen B, Sivonen K, Smith L, Stein T, Sussmuth RD, Tagg JR, Tang G-L, Truman AW, Vederas JC, Walsh CT, Walton JD, Wenzel SC, Willey JM, and van der Donk WA. 2013. Ribosomally synthesized and posttranslationally modified peptide natural products: overview and recommendations for a universal nomenclature. Natural Product Reports 30:108-160. 10.1039/C2NP20085F

Bäckhed F, Ley RE, Sonnenburg JL, Peterson DA, and Gordon JI. 2005. Host-Bacterial Mutualism in the Human Intestine. Science 307:1915-1920. 10.1126/science.1104816

Belstrøm D, Holmstrup P, Bardow A, Kokaras A, Fiehn N-E, and Paster BJ. 2016. Temporal Stability of the Salivary Microbiota in Oral Health. PLOS ONE 11:e0147472. 10.1371/journal.pone.0147472

Carver T, Harris SR, Berriman M, Parkhill J, and McQuillan JA. 2012. Artemis: an integrated platform for visualization and analysis of high-throughput sequence-based experimental data. Bioinformatics 28:464-469. 10.1093/bioinformatics/btr703

Clemente Jose C, Ursell Luke K, Parfrey Laura W, and Knight R. 2012. The Impact of the Gut Microbiota on Human Health: An Integrative View. Cell 148:1258-1270. http://dx.doi.org/10.1016/j.cell.2012.01.035

Corr SC, Li Y, Riedel CU, O'Toole PW, Hill C, and Gahan CGM. 2007. Bacteriocin production as a mechanism for the antiinfective activity of Lactobacillus salivarius UCC118. Proceedings of the National Academy of Sciences 104:7617-7621. 10.1073/pnas.0700440104

Delcher AL, Harmon D, Kasif S, White O, and Salzberg SL. 1999. Improved microbial gene identification with GLIMMER. Nucleic Acids Res 27:4636-4641.

Donia Mohamed S, Cimermancic P, Schulze Christopher J, Wieland Brown Laura C, Martin J, Mitreva M, Clardy J, Linington Roger G, and Fischbach Michael A. 2014. A Systematic Analysis of Biosynthetic Gene Clusters in the Human Microbiome Reveals a Common Family of Antibiotics. Cell 158:1402-1414. 10.1016/j.cell.2014.08.032 
427

428

429

430

431

432

433

434

435

436

437

438

439

440

441

442

443

444

445

446

447

448

449

450

451

452

453

454

455

456

457

458

459

460

461

462

463

464

465

466

467

468

Dusko Ehrlich S. 2010. [Metagenomics of the intestinal microbiota: potential applications]. Gastroenterol Clin Biol 34 Suppl 1:S23-28. 10.1016/s0399-8320(10)70017-8

Edgar RC. 2004. MUSCLE: multiple sequence alignment with high accuracy and high throughput. Nucleic Acids Res 32:1792-1797. 10.1093/nar/gkh340

Erejuwa OO, Sulaiman SA, and Ab Wahab MS. 2014. Modulation of gut microbiota in the management of metabolic disorders: the prospects and challenges. Int J Mol Sci 15:4158-4188. 10.3390/ijms15034158

Faith JJ, Guruge JL, Charbonneau M, Subramanian S, Seedorf H, Goodman AL, Clemente JC, Knight R, Heath AC, Leibel RL, Rosenbaum M, and Gordon JI. 2013. The Long-Term Stability of the Human Gut Microbiota. Science 341. 10.1126/science.1237439

Flint HJ, Scott KP, Louis P, and Duncan SH. 2012. The role of the gut microbiota in nutrition and health. Nat Rev Gastroenterol Hepatol 9:577-589.

Gales M, and Young S. 2007. The application of hidden Markov models in speech recognition. Found Trends Signal Process 1:195-304. 10.1561/2000000004

Garg N, Salazar-Ocampo LMA, and van der Donk WA. 2013. In vitro activity of the nisin dehydratase NisB. Proceedings of the National Academy of Sciences of the United States of America 110:7258-7263. 10.1073/pnas.1222488110

Goto Y, Li B, Claesen J, Shi Y, Bibb MJ, and van der Donk WA. 2010. Discovery of Unique Lanthionine Synthetases Reveals New Mechanistic and Evolutionary Insights. PLoS Biol 8:e1000339. 10.1371/journal.pbio.1000339

Jeffery IB, Lynch DB, and O'Toole PW. 2016. Composition and temporal stability of the gut microbiota in older persons. ISME J 10:170-182. 10.1038/ismej.2015.88

Kelly WL, Pan L, and Li C. 2009. Thiostrepton biosynthesis: prototype for a new family of bacteriocins. J Am Chem Soc 131:4327-4334. 10.1021/ja807890a

Madera M, and Gough J. 2002. A comparison of profile hidden Markov model procedures for remote homology detection. Nucleic Acids Res 30:4321-4328.

Menzel P, Ng KL, and Krogh A. 2016. Fast and sensitive taxonomic classification for metagenomics with Kaiju. Nature Communications 7:11257. 10.1038/ncomms11257

http://www.nature.com/articles/ncomms11257\#supplementary-information

Moroni O, Kheadr E, Boutin Y, Lacroix C, and Fliss I. 2006. Inactivation of Adhesion and Invasion of Food-Borne Listeria monocytogenes by Bacteriocin-Producing Bifidobacterium Strains of Human Origin. Applied and Environmental Microbiology 72:6894-6901. 10.1128/aem.00928-06

Morton JT, Freed SD, Lee SW, and Friedberg I. 2015. A large scale prediction of bacteriocin gene blocks suggests a wide functional spectrum for bacteriocins. BMC Bioinformatics 16:1-9. 10.1186/s12859-015-0792-9

Ortega MA, Hao Y, Zhang Q, Walker MC, van der Donk WA, and Nair SK. 2015. Structure and mechanism of the tRNA-dependent lantibiotic dehydratase NisB. Nature 517:509-512. 10.1038/nature13888

Park J, Karplus K, Barrett C, Hughey R, Haussler D, Hubbard T, and Chothia C. 1998. Sequence comparisons using multiple sequences detect three times as many remote homologues as pairwise methods. J Mol Biol 284:1201-1210. 10.1006/jmbi.1998.2221 
469

470

471

472

473

474

475

476

477

478

479

480

481

482

483

484

485

486

487

488

489

490

491

492

493

494

495

496

497

498

499

500

501

502

503

504

505

506

507

508

509

510

511

Perez RH, Zendo T, and Sonomoto K. 2014. Novel bacteriocins from lactic acid bacteria (LAB): various structures and applications. Microbial Cell Factories 13:S3-S3. 10.1186/14752859-13-S1-S3

Punta M, Coggill PC, Eberhardt RY, Mistry J, Tate J, Boursnell C, Pang N, Forslund K, Ceric G, Clements J, Heger A, Holm L, Sonnhammer ELL, Eddy SR, Bateman A, and Finn RD. 2012. The Pfam protein families database. Nucleic Acids Res 40:D290-D301. 10.1093/nar/gkr1065

Qin J, Li R, Raes J, Arumugam M, Burgdorf KS, Manichanh C, Nielsen T, Pons N, Levenez F, Yamada T, Mende DR, Li J, Xu J, Li S, Li D, Cao J, Wang B, Liang H, Zheng H, Xie Y, Tap J, Lepage $P$, Bertalan M, Batto J-M, Hansen T, Le Paslier D, Linneberg A, Nielsen HB, Pelletier E, Renault P, Sicheritz-Ponten T, Turner K, Zhu H, Yu C, Li S, Jian M, Zhou Y, Li Y, Zhang X, Li S, Qin N, Yang H, Wang J, Brunak S, Dore J, Guarner F, Kristiansen K, Pedersen O, Parkhill J, Weissenbach J, Bork P, Ehrlich SD, and Wang J. 2010. A human gut microbial gene catalogue established by metagenomic sequencing. Nature 464:5965. http://www.nature.com/nature/journal/v464/n7285/suppinfo/nature08821 S1.html

R Core Team. 2015. R: A Language and Environment for Statistical Computing.

Rea MC, Dobson A, O'Sullivan O, Crispie F, Fouhy F, Cotter PD, Shanahan F, Kiely B, Hill C, and Ross RP. 2011a. Effect of broad- and narrow-spectrum antimicrobials on Clostridium difficile and microbial diversity in a model of the distal colon. Proceedings of the National Academy of Sciences 108:4639-4644. 10.1073/pnas.1001224107

Rea MC, Ross RP, Cotter PD, and Hill C. 2011b. Classification of Bacteriocins from Gram-Positive Bacteria. In: Drider D, and Rebuffat S, eds. Prokaryotic Antimicrobial Peptides: From Genes to Applications. New York, NY: Springer New York, 29-53.

Seifert M, Abou-El-Ardat K, Friedrich B, Klink B, and Deutsch A. 2014. Autoregressive HigherOrder Hidden Markov Models: Exploiting Local Chromosomal Dependencies in the Analysis of Tumor Expression Profiles. PLOS ONE 9:e100295. 10.1371/journal.pone.0100295

Sender R, Fuchs S, and Milo R. 2016. Revised estimates for the number of human and bacteria cells in the body. bioRxiv. 10.1101/036103

Sherlock C, Xifara T, Telfer S, and Begon M. 2013. A coupled hidden Markov model for disease interactions. Journal of the Royal Statistical Society Series C, Applied Statistics 62:609627. 10.1111/rssc.12015

Skewes-Cox P, Sharpton TJ, Pollard KS, and DeRisi JL. 2014. Profile Hidden Markov Models for the Detection of Viruses within Metagenomic Sequence Data. PLoS ONE 9:e105067. 10.1371/journal.pone.0105067

Stein RR, Bucci V, Toussaint NC, Buffie CG, Rätsch G, Pamer EG, Sander C, and Xavier JB. 2013. Ecological Modeling from Time-Series Inference: Insight into Dynamics and Stability of Intestinal Microbiota. PLoS Comput Biol 9:e1003388. 10.1371/journal.pcbi.1003388

The Human Microbiome Project Consortium. 2012. Structure, function and diversity of the healthy human microbiome. Nature 486:207-214. 10.1038/nature11234

Upton M, Tagg JR, Wescombe P, and Jenkinson HF. 2001. Intra- and Interspecies Signaling between Streptococcus salivarius and Streptococcus pyogenes Mediated by SalA and 
512

513

514

515

516

517

518

519

520

521

522

523

524

525

526

527

528

529

530

531

532

533

534

535

536

SalA1 Lantibiotic Peptides. J Bacteriol 183:3931-3938. 10.1128/JB.183.13.39313938.2001

van Heel AJ, de Jong A, Montalban-Lopez M, Kok J, and Kuipers OP. 2013. BAGEL3: Automated identification of genes encoding bacteriocins and (non-)bactericidal posttranslationally modified peptides. Nucleic Acids Res 41:W448-453. 10.1093/nar/gkt391

Walsh CJ, Guinane CM, Hill C, Ross RP, O'Toole PW, and Cotter PD. 2015. In silico identification of bacteriocin gene clusters in the gastrointestinal tract, based on the Human Microbiome Project's reference genome database. BMC Microbiol 15:183. 10.1186/s12866-015-0515-4

Walsh CJ, Guinane CM, O'Toole PW, and Cotter PD. 2014. Beneficial modulation of the gut microbiota. FEBS Lett 588:4120-4130. 10.1016/j.febslet.2014.03.035

Weber T, Blin K, Duddela S, Krug D, Kim HU, Bruccoleri R, Lee SY, Fischbach MA, Müller R, Wohlleben W, Breitling R, Takano E, and Medema MH. 2015. antiSMASH 3.0-a comprehensive resource for the genome mining of biosynthetic gene clusters. Nucleic Acids Res 43:W237-W243. 10.1093/nar/gkv437

Xie L, and van der Donk WA. 2004. Post-translational modifications during lantibiotic biosynthesis. Curr Opin Chem Biol 8:498-507. 10.1016/j.cbpa.2004.08.005

Yan X, Gurtler JB, Fratamico PM, Hu J, and Juneja VK. 2012. Phylogenetic identification of bacterial MazF toxin protein motifs among probiotic strains and foodborne pathogens and potential implications of engineered probiotic intervention in food. Cell \& Bioscience 2:1-13. 10.1186/2045-3701-2-39

Yoon B-J. 2009. Hidden Markov Models and their Applications in Biological Sequence Analysis. Current Genomics 10:402-415. 10.2174/138920209789177575

Zheng J, Gänzle MG, Lin XB, Ruan L, and Sun M. 2014. Diversity and dynamics of bacteriocins from human microbiome. Environ Microbiol:n/a-n/a. 10.1111/1462-2920.12662 


\section{Table $\mathbf{1}$ (on next page)}

Controls used in validation of the profile HMM, listing the lantibiotic produced and the subclass of modification protein responsible for lanthionine dehydration for each strain.

${ }^{a}$ Lanthionine dehydratase protein identified by our model

${ }^{\mathrm{b}}$ Lanthionine dehydratase protein identified by PF04738 model

${ }^{c}$ Lanthionine dehydratase protein identified by BlastP

${ }^{d}$ Lanthionine dehydratase protein identified by BAGEL3 


\begin{tabular}{|c|c|c|}
\hline Strain & Bacteriocin & Subclass \\
\hline Lactococcus lactis ssp. lactis S0 a,b,c,d & Nisin Z & LanB \\
\hline Lactococcus lactis ssp. lactis CV56 $6^{\mathrm{a}, \mathrm{b}, \mathrm{c}, \mathrm{d}}$ & Nisin A & LanB \\
\hline Lactococcus lactis ssp. lactis $1 \mathrm{O}-1 \mathrm{a}, \mathrm{b}, \mathrm{c}, \mathrm{d}$ & Nisin Z & LanB \\
\hline Bacillus subtilis subsp. spizizienii ATCC $6633^{a, b, c, d}$ & Subtilin & LanB \\
\hline Staphylococcus aureus subsp. aureus USA300_FPR3757 a,c,d & Bsa & LanB \\
\hline Streptococcus mutans $\mathrm{CH} 43^{\mathrm{a}, \mathrm{b}, \mathrm{d}}$ & Mutacin I & LanB \\
\hline Streptococcus mutans UA787 a,b,d & Mutacin III & LanB \\
\hline Streptococcus pyogenes ${ }^{\mathrm{a}, \mathrm{b}, \mathrm{c}, \mathrm{d}}$ & Streptin & LanB \\
\hline Staphylococcus epidermidis ${ }^{\mathrm{a}, \mathrm{b}, \mathrm{c}, \mathrm{d}}$ & Pep5 & LanB \\
\hline Lactococcus lactis subsp. lactis KF147 c & None & - \\
\hline Streptococcus mutans GS-5 & Mutacin GS-5 & LanM \\
\hline Lactococcus lactis subsp. lactis plasmid pES2 & Lacticin 481 & LanM \\
\hline Streptomyces cinnamoneus cinnamoneus DSM 4005 & Cinnamycin & LanM \\
\hline Bacillus paralichenformis APC 1576 & Formicin & LanM \\
\hline Streptococcus salivarius plasmid pSsal-K12 & Salivaricin B & LanM \\
\hline Streptomyces venezuelae ATCC $10712^{d}$ & Venezuelin & LanL \\
\hline
\end{tabular}


Table 2 (on next page)

Number of metagenomic samples per body site screened. 


\begin{tabular}{lr}
\hline Site & Number of Samples \\
\hline Attached Keratinized Gingiva & 6 \\
Buccal Mucosa & 107 \\
Palatine Tonsils & 6 \\
Saliva & 3 \\
Stool & 139 \\
Subgingival Plaque & 7 \\
Supragingival Plaque & 118 \\
Throat & 7 \\
Tongue Dorsum & 128
\end{tabular}

1 


\section{Table 3(on next page)}

Detailed information of all lanthionine dehydratase proteins identified in the gastrointestinal tract subset of the Human Microbiome Project's reference genome database using our profile HMM. 


\begin{tabular}{llr}
\hline Accession & Strain & E Value \\
\hline JH414709 & Bacillus sp. 7_6_55CFAA_CT2 & $9.0 \mathrm{E}-16$ \\
GL636578 & Coprobacillus sp. 29_1 & $3.7 \mathrm{E}-67$ \\
AKCB01000002 Coprobacillus sp. D6 & $4.5 \mathrm{E}-68$ \\
JH126516 & Dorea formicigenerans 4_6_53AFAA & \\
ACEP01000029 Eubacterium hallii DSM3353 & $2.3 \mathrm{E}-81$ \\
KI391961 & Fusobacterium nucleatum subsp. animalis 3_1_33 & $2.2 \mathrm{E}-09$
\end{tabular}

1 


\section{Figure 1}

BAGEL3 output of putative bacteriocin gene clusters identified in the positive controls used for validation of our new profile HMM.

Each predicted open reading frame is colour-coded based on the role it plays in lantibiotic biosynthesis.

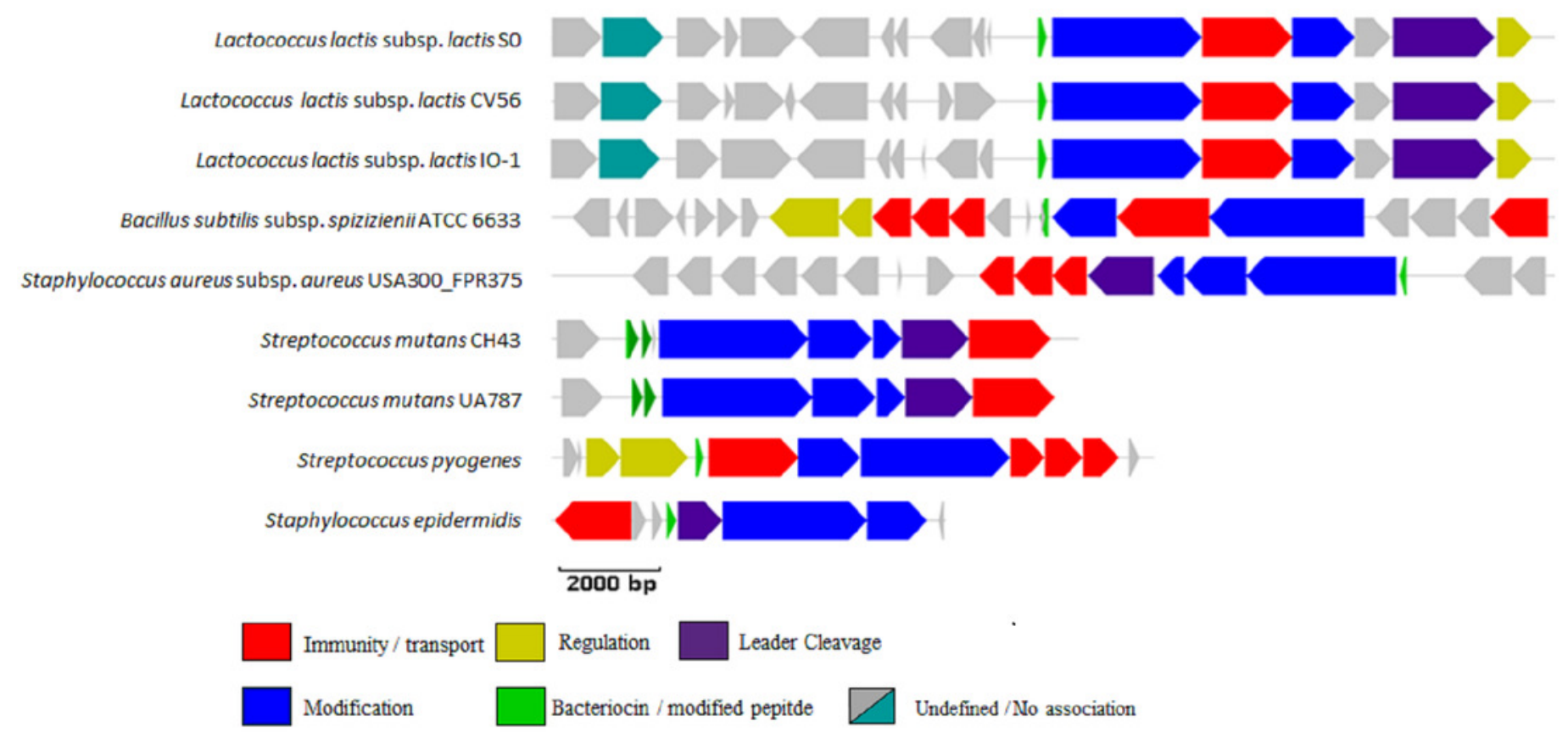




\section{Figure 2}

Barchart depicting the distribution of lanthionine dehydratase protein numbers identified by our new profile HMM in metagenomic samples from the stool and oral microbiota.

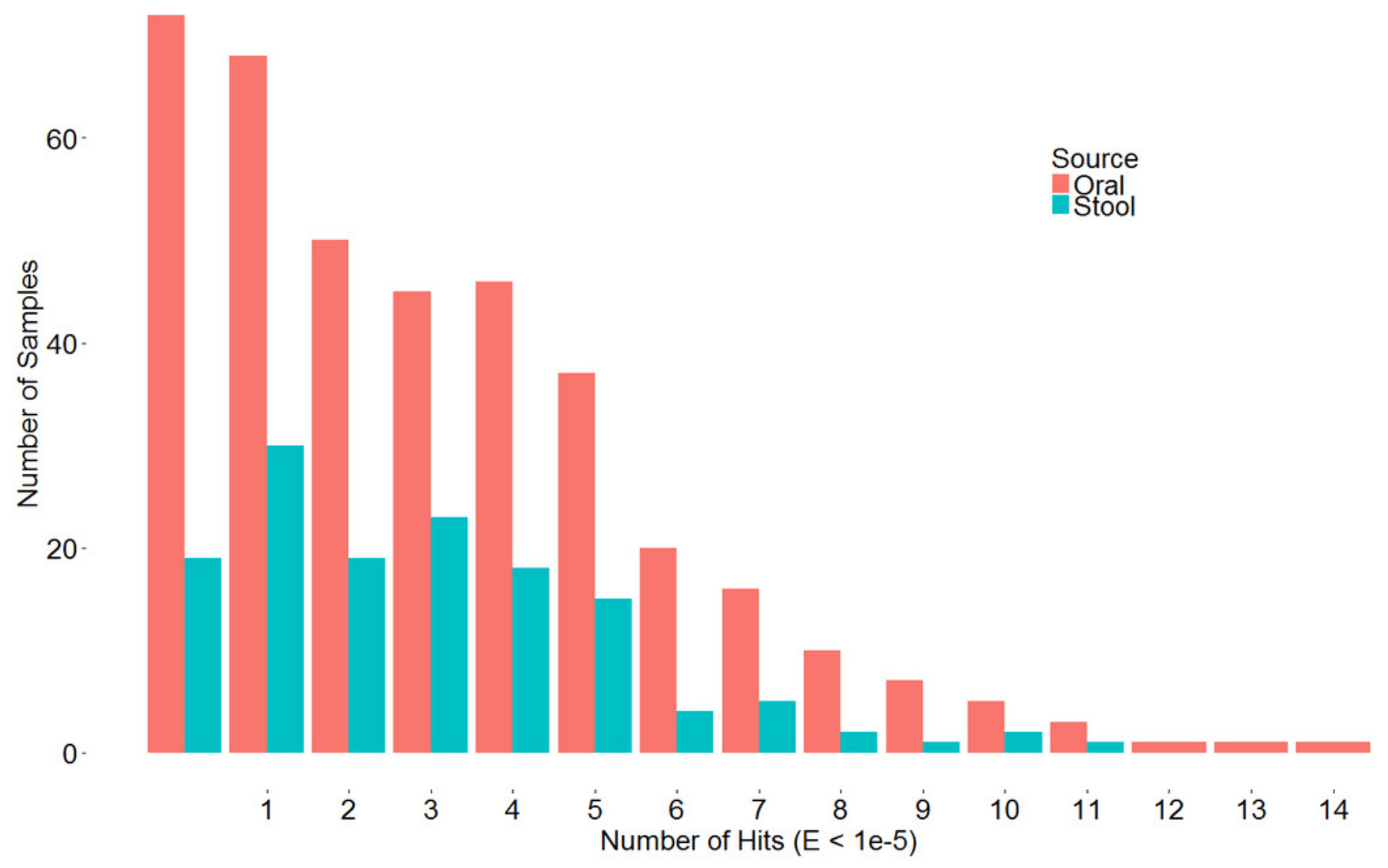


Figure 3

Venn diagram illustrating the numbers of lanthionine dehydratase proteins reported in stool (A) and oral (B) metagenomic data by single and multiple methods. 
A
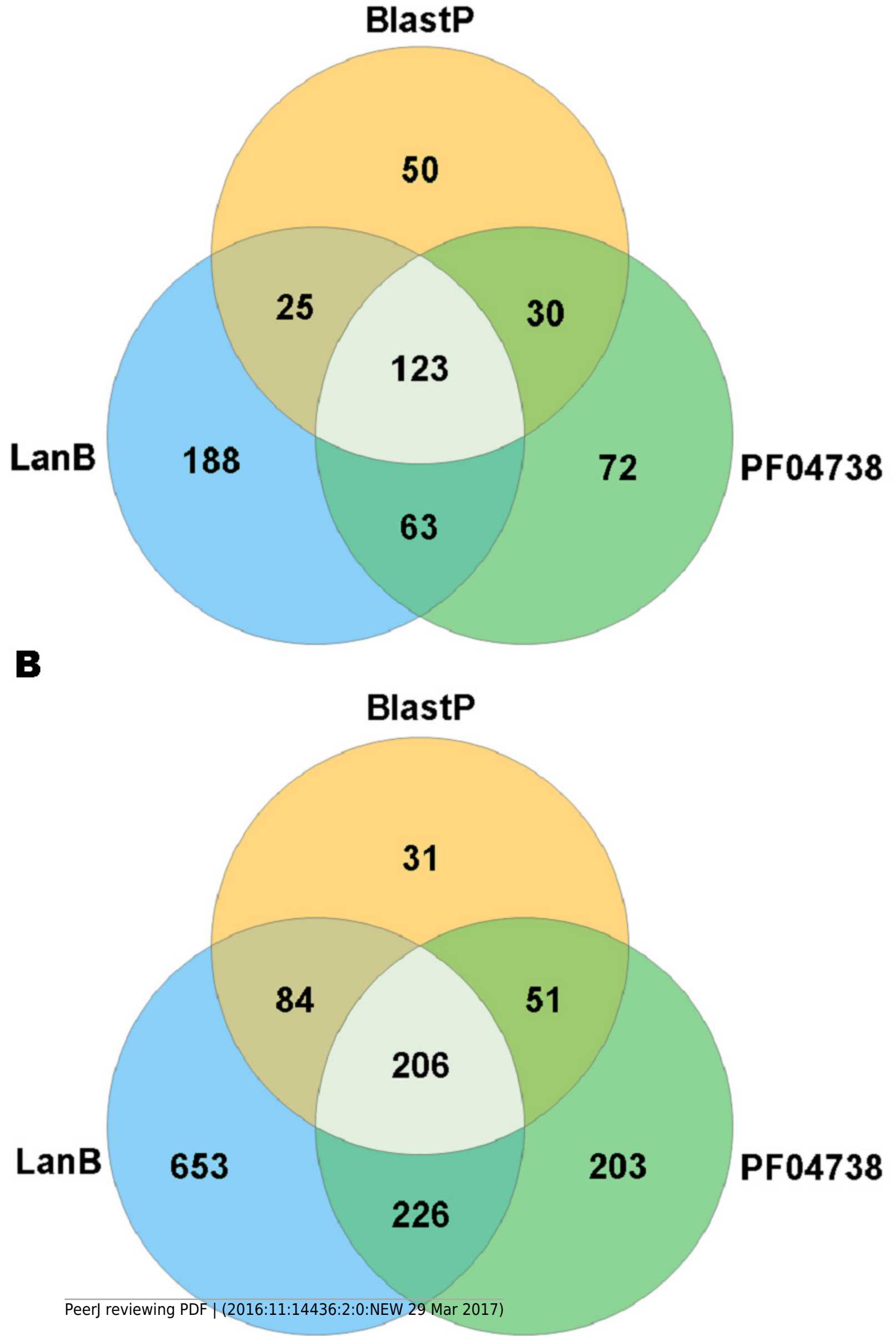
Figure 4

Comparison of lanthionine dehydratase density by body site reported by all three methods

Insert shows overall comparison between stool and oral environments.

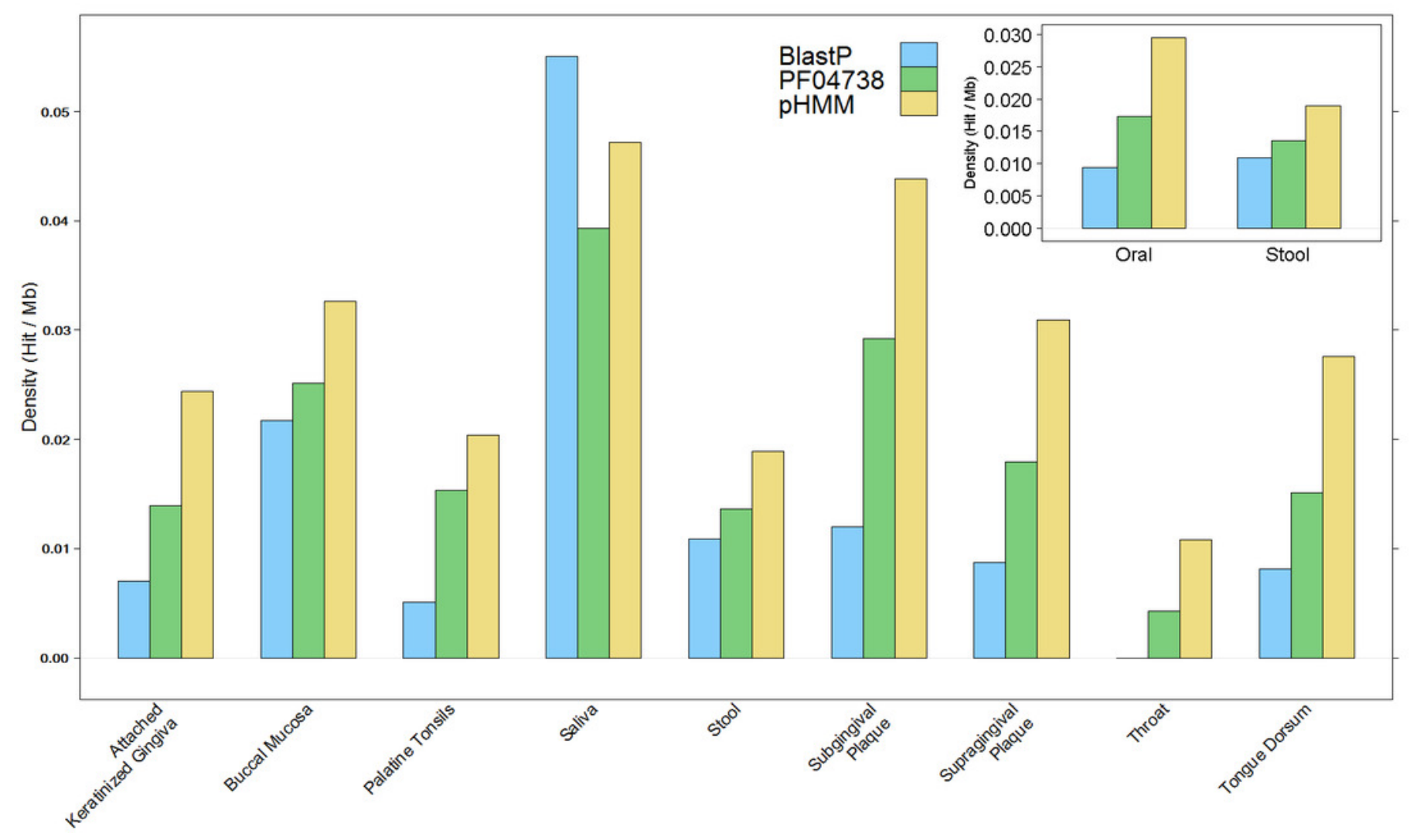




\section{Figure 5}

BAGEL3 output of three putative bacteriocin gene clusters identified from the gastrointestinal tract subset of the Human Microbiome Project's reference genome database by our new profile HMM.

A - Coprobacillus sp. D6

B - Coprobacillus sp. 29_1

C - Dorea formicigenerans 4_6_53AFAA

Each predicted open reading frame is colour-coded based on the role it plays in lantibiotic biosynthesis.

$\mathbf{A}$

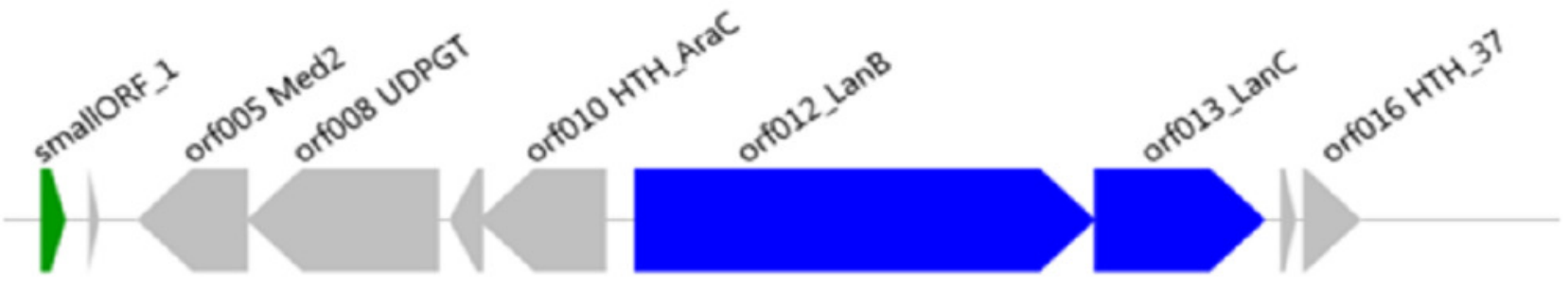

B

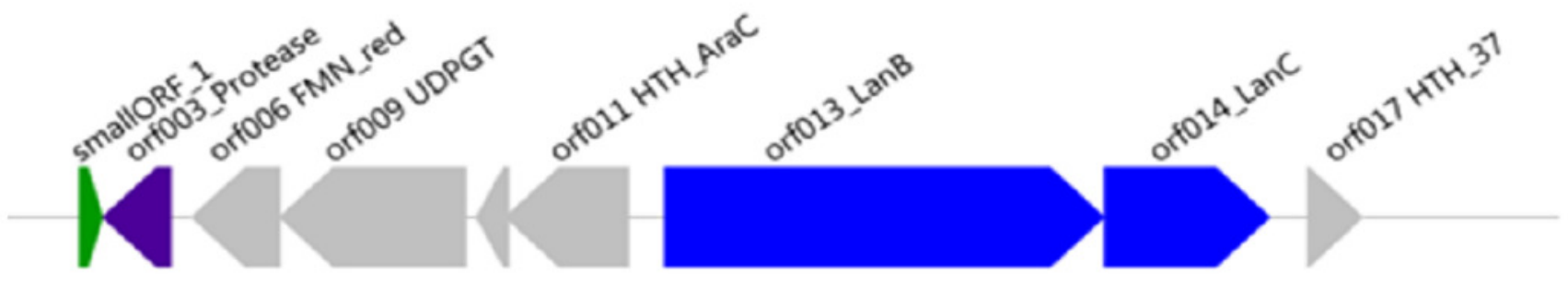

C

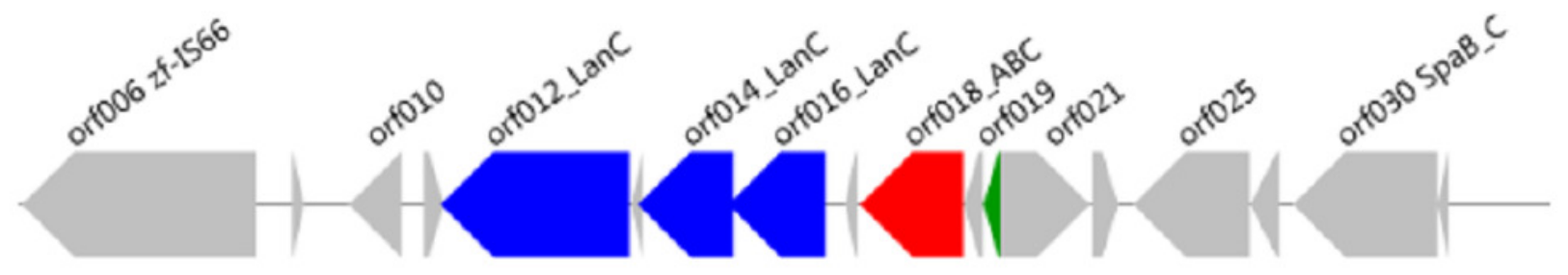

Immunity / transport

Leader Cleavage

Modification 